\title{
WIMP annihilation and cooling of neutron stars
}

\author{
Chris Kouvaris* \\ CERN Theory Division, CH-1211 Geneva 23, Switzerland, \\ University of Southern Denmark, Campusvej 55, DK-5230 Odense, Denmark \\ and The Niels Bohr Institute, Blegdamsvej 17, DK-2100 Copenhagen, Denmark
}

(Received 27 August 2007; published 28 January 2008)

\begin{abstract}
We study the effect of WIMP annihilation on the temperature of a neutron star. We shall argue that the released energy due to WIMP annihilation inside the neutron stars might affect the temperature of stars older than $10 \times 10^{6}$ years, flattening out the temperature at $\sim 10^{4} \mathrm{~K}$ for a typical neutron star.
\end{abstract}

PACS numbers: 97.60.Jd

\section{INTRODUCTION}

Since Zwicky proposed the problem of the "missing mass" in 1933, a lot of theoretical and experimental effort has been made in order to unveil the nature of dark matter. Today, WMAP has provided very accurate data regarding the matter density in the Universe [1]. The energy density of the Universe is composed of $4 \%$ atoms and roughly $22 \%$ dark matter. Data from recent observations indicate that dark matter cannot be attributed more than $20 \%$ to dim objects like black holes, brown dwarfs, and giant planets [2]. From a theoretical point of view, several candidates rise from different theories, such as neutralinos [3,4], Majorana neutrinos, and, lately, technibaryons provided by theories that are not ruled out by the electroweak precision measurements [5-10]. From the experimental point of view, the focus is on the direct and indirect detection of dark matter particles. The direct detection might occur in underground experiments like CDMS that, in principle, can detect recoil energies from collisions between weakly interacting massive particles (WIMPs) and nuclei, or atmospheric experiments like the X-ray Quantum Calorimeter (XQC), where strongly interacting particles might collide with the detector. The indirect detection might occur via gamma-ray and neutrino telescopes, where the presence of WIMPs can be detected indirectly, by observing products of WIMP annihilations. In particular, provided that WIMPs can annihilate and because they can be trapped inside the Earth or the sun, such annihilations would produce jets of particles and more specifically neutrinos coming straight from the center of the Earth or the sun, that could possibly be detected by neutrino telescopes [11-13]. On the other hand, gammaray telescopes can, in principle, detect gamma rays produced by WIMP annihilation at the center of the Galaxy [14]. Both direct and indirect detection experiments can impose strong constraints on the cross section of the WIMP with the nuclei. For instance, heavy Dirac neutrinos have been excluded as WIMPs for masses up to several $\mathrm{TeV}$, because their elastic cross section with nuclei is suffi-

*kouvaris@nbi.dk ciently large and therefore they should have been detected by now in CDMS [15].

In this paper we investigate the possibility of a different kind of indirect signature of WIMP annihilation. Instead of looking at the indirect signals from the annihilation of trapped WIMPs inside the Earth or the sun, we examine the consequences of WIMP annihilation on the temperature of neutron stars. The neutron stars are massive compact objects with very low temperatures. Naively one might expect that, since the mass of the trapped WIMPs inside a neutron star represents a tiny fraction of the overall mass of the star, such an effect should be negligible. However, the annihilation of massive particles inside the star releases a huge amount of energy that is heating up the star. As we shall argue, once the accretion rate of dark matter particles equilibrates the rate of annihilation, the amount of released energy is independent of the star's temperature, and therefore at late times the WIMP annihilation can keep the star at a constant temperature that depends on the mass and the radius of the star, the cross section of annihilation, and the local dark matter density of the star.

The paper is organized as follows: First we calculate the rate of dark matter accretion onto the neutron star including general relativity corrections. Then we calculate the annihilation rate for the WIMPs, and we calculate the effect of the WIMP annihilation on the cooling curves of a typical neutron star made of regular nuclear matter. We present our conclusions in the last section.

\section{WIMP'S ACCRETION RATE ONTO THE NEUTRON STAR}

The accretion of dark matter particles inside the Earth and the sun is not a new subject. Press and Spergel studied first in [11] the capture rate of WIMPs inside the Earth and the sun. More elaborate calculations were also done by Gould [12,13], taking into account several effects specifically for the case of the Earth and the sun. An estimate of the accretion rate onto a neutron star was also provided by Goldman and Nussinov [16], who were the first to study effects of WIMPs on neutron stars. In this section we calculate the accretion rate of WIMPs onto a typical neu- 
tron star including also general relativity corrections that turn out to affect the rate up to $70 \%$. Our derivation is along the lines of [11]. We assume that the WIMP population has a Maxwell-Boltzmann distribution of velocities,

$$
p(v) d v=n_{0}\left(\frac{3}{2 \pi \bar{v}^{2}}\right)^{3 / 2} 4 \pi v^{2} \exp \left(\frac{-3 v^{2}}{2 \bar{v}^{2}}\right) d v,
$$

where $\bar{v}=270 \mathrm{~km} / \mathrm{s}$, and $n_{0}$ is the number density of the WIMPs in the neighborhood of the neutron star. The flux of WIMPs (number per area per time) that crosses a spherical surface of radius $R$, with velocity between $v$ and $v+d v$ and angle with respect to the normal between $\theta$ and $\theta+$ $d \theta$, is

$$
d F=n_{0}\left(\frac{3}{2 \pi \bar{v}^{2}}\right)^{3 / 2} \pi v^{3} \exp \left(\frac{-3 v^{2}}{2 \bar{v}^{2}}\right) d\left(\cos ^{2} \theta\right) d v .
$$

We can express the flux in a more convenient way with respect to the two invariants of the motion, i.e. the energy of the WIMP per mass $E=(1 / 2) v^{2}$ and the angular momentum per mass $J=v R \sin \theta$. The total accretion rate (number of particles per time) is [11]

$$
d \mathcal{F}=4 \pi R^{2} d F=n_{0}\left(\frac{3}{2 \pi \bar{v}^{2}}\right)^{3 / 2} \exp \left(\frac{-3 E}{\bar{v}^{2}}\right) 4 \pi^{2} d E d J^{2}
$$

The actual capture rate of WIMPs by the star can be calculated in two steps. The first one is to determine what part of the phase space for $E$ and $J$ can give orbits for the WIMPs that intersect with the neutron star. In the second step we have to determine what fraction of the particles that intersect with the star lose enough energy so they can be trapped inside the star. For the first part of the calculation, we have to find the trajectories that have a perihelion (closest distance to the center of the star) at most equal to the radius of the star. Press and Spergel calculated this using classical Newtonian mechanics. The perihelion is

$$
r_{\text {peri }}=\left(\frac{J^{2}}{G M}\right) /\left(1+\sqrt{1+2 \frac{J^{2}}{G M} \frac{E}{G M}}\right),
$$

where $G$ is the gravitational constant and $M$ is the mass of the neutron star. This expression has two limiting cases. For $J^{2} E \ll(G M)^{2}$

$$
r_{\text {peri }}=\frac{J^{2}}{2 G M},
$$

and for $J^{2} E \gg(G M)^{2}$

$$
r_{\text {peri }}=\sqrt{\frac{J^{2}}{2 E}}
$$

The two regimes are separated by the hyperbola

$$
\frac{J^{2}}{G M} \frac{E}{G M}=1 \text {. }
$$

Since Eq. (3) falls exponentially with respect to the energy, we approximate (as it is done in [11]) the exponential as unity with $E$ varying from zero to $(1 / 3) \bar{v}^{2}$, which is the characteristic scale of the exponential. In addition, $E$ is also restricted to values smaller than $E_{0}$, where $E_{0}$ represents a constant that parametrizes the maximum kinetic energy per mass of the WIMP at an asymptotically large distance from the star in order for the WIMP to be captured by the star. We shall determine $E_{0}$ later on. Therefore, as it was argued in [11], the accretion rate of capturable WIMPs is given by (3), if we integrate over $E$ from zero to the minimum between $(1 / 3) \bar{v}^{2}$ and $E_{0}$ and over $J^{2}$ from zero up to $2 G M R$ [that comes from (5) if $r_{\text {peri }}=R$ ], where $R$ is now the radius of the star. The rate can be written as

$$
\mathcal{F}=n_{0}\left(\frac{3}{2 \pi \bar{v}^{2}}\right)^{3 / 2} 4 \pi^{2}(2 G M R) \min \left(\frac{1}{3} \bar{v}^{2}, E_{0}\right) .
$$

This formula differs by a factor of 2 with respect to the corresponding one in [11], as it was first pointed out by Gould [12]. Although the above formula is a good approximate relation for the capture rate of WIMPs for the sun and the Earth, for the case of a neutron star, general relativity corrections increase the rate significantly. To show this, we are going to use the timelike geodesic equations that describe the motion of a particle in a Schwarzschild metric. The trajectory for nonrelativistic particles (such as the WIMPs) is given by [17]

$$
\left(\frac{d u}{d \phi}\right)^{2}=2 m u^{3}-u^{2}+\frac{2}{J^{2}} m u+\frac{2 E}{J^{2}},
$$

where $m=G M$ (in natural units) and $u=1 / r$. We want to find for what values of the phase space of $E$ and $J^{2}$ the perihelion becomes smaller or equal to the radius of the neutron star. At the perihelion $d u / d \phi=0$ and $u=1 / R$ (for $r_{\text {peri }}=R$ ). If we express $E$ in units of $G M / R$ and $J^{2}$ in units of $G M R$, Eq. (9) gives

$$
E=\frac{1}{2}\left(1-\frac{2 G M}{R}\right) J^{2}-1 .
$$

The above equation gives the relation between $E$ and $J$ in order for the perihelion to be $R$. For $E=0, J^{2}=2 /(1-$ $2 G M / R)$. This means that the allowed phase space for $J^{2}$ has increased compared to the Newtonian case from 2 (again in units of $G M R)$ to $2 /(1-2 G M / R)$. For a typical neutron star of mass 1.4 the solar mass $M_{\odot}$ and a radius of $10 \mathrm{~km}, J^{2}=2 /(1-2 G M / R) \simeq 3.4$. This is a $70 \%$ increase in the phase space of $J^{2}$ and the capture rate compared to the classical case. Therefore Eq. (8) should be modified as

$$
\begin{aligned}
\mathcal{F}= & n_{0}\left(\frac{3}{2 \pi \bar{v}^{2}}\right)^{3 / 2} 4 \pi^{2}(2 G M R) \frac{1}{1-2 G M / R} \\
& \times \min \left(\frac{1}{3} \bar{v}^{2}, E_{0}\right) .
\end{aligned}
$$


Now we estimate $E_{0}$. We shall show that, in the case of a neutron star, $E_{0} \gg(1 / 3) \bar{v}^{2}$, and therefore Eq. (11) should be always taken with $(1 / 3) \bar{v}^{2}$ as the minimum. A WIMP that intersects with the neutron star might or might not interact with the nuclear matter inside the star. If it does scatter at some point, the recoil energy and the energy loss of the particle are $0<T<4 m_{n} m_{\chi} /\left(m_{n}+m_{\chi}\right)^{2}$, where $T$ is the recoil energy and $m_{n}$ and $m_{\chi}$ are the masses of the nucleus and the mass of the WIMP, respectively. If we assume that the scattering is isotropic, then the recoil energy should be uniformly distributed. The condition that holds in order to capture a WIMP is that the energy loss in the scattering should be at least equal to the initial kinetic energy of the WIMP at an asymptotically large distance from the star. If this condition is fulfilled, the WIMP stays in a bound state with the star. Therefore, for an average collision that takes place in the star, this condition can be written as

$$
\Delta E=\frac{2 m_{n} m_{\chi}}{\left(m_{n}+m_{\chi}\right)^{2}}\left(1-\sqrt{1-\frac{2 G M}{R}}\right) \geq E_{0}
$$

where we took into account the gravitational redshift effect. Again we have chosen to set $c=1$. If we plug the typical values we used before for the mass and the radius of a neutron star and a WIMP mass of the order of $\mathrm{TeV}$, we find that $E_{0}$ is 3 orders of magnitude larger than $(1 / 3) \bar{v}^{2}$, and therefore, for all the cases of interest, we can use (11) with $(1 / 3) \bar{v}^{2}$ as the minimum.

Equation (11) gives the rate of capturable WIMPs, that is, the number of WIMPs per second that intersect with the neutron star. However, as mentioned before, in order for the WIMP to be trapped in the star, one or more collisions have to take place. We know from classical mechanics that, if the WIMP does not scatter while traveling through the star, it cannot be captured by the star. We calculate now the fraction of the capturable WIMPs [given by (11)] that can yield scatters into bound orbits. Such a fraction would depend strongly on the elastic scattering cross section of the WIMP-nucleus system. For the typical neutron star of mass $1.4 M_{\odot}$ and $R=10 \mathrm{~km}$, the average density of neutrons is $\rho=3 M /\left(4 \pi R^{3} m_{n}\right) \simeq 4 \times 10^{38}$ neutrons $/ \mathrm{cm}^{3}$. If we take a typical value for the elastic cross section between WIMP-neutron of the order of $10^{-44} \mathrm{~cm}^{2}$, the mean free path is about $1 \mathrm{~km}$. Since, for an average WIMP, even one scattering is enough to result in a bound orbit around the star, a mean free path of $1 \mathrm{~km}$ means that the fraction of the capturable WIMPs that will be trapped is very close to 1 . To entertain this, if for simplicity we assume that the WIMP has a straight trajectory while inside the neutron star, a segment of $1 \mathrm{~km}$ corresponds to an impact parameter of $9.9 \mathrm{~km}$, which means that only if the WIMP intersects between 9.9 and $10 \mathrm{~km}$ from the center of the star will it travel a distance less than $1 \mathrm{~km}$ inside the star. Obviously if the cross section is larger than $10^{-44} \mathrm{~cm}^{2}$, the fraction saturates even faster to 1 . We shall give now a more quantitative answer about the fraction following the derivation of [11]. The fraction $f$ of the particles that undergo one or more scatterings while inside the star is defined as

$$
f=\left\langle 1-\exp \left[-\int \frac{\sigma_{\chi} \rho}{m_{n}} d l\right]\right\rangle \simeq\left\langle\int \frac{\sigma_{\chi} \rho}{m_{n}} d l\right\rangle,
$$

where the last approximation holds if the elastic cross section between WIMP-nucleus $\sigma_{\chi}$ is smaller than $\sigma_{\text {crit }}=$ $m_{n} R^{2} / M \simeq 6 \times 10^{-46} \mathrm{~cm}^{2}$. Equation (13) now reads

$$
f \simeq \frac{\sigma_{\chi}}{\sigma_{\text {crit }}}\left\langle\int \frac{\rho}{M / R^{3}} \frac{d l}{R}\right\rangle .
$$

In order to find $f$, we examine trajectories with $E=0$ as in [11], since $E \ll G M / R$. We average over $J^{2}$ (which ranges from 0 to 3.4 for our typical neutron star). For an accurate calculation of $f$, we need to know the exact density profile of the star, in order to know explicitly the mass of the star $M(r)$ as a function of the radius. Here we give an estimate of $f$, by assuming for simplicity that the density of the star is constant through the whole volume. This means that $M(r) / M=(r / R)^{3}$ (where $M$ is the total mass of the star). If we take the derivative of Eq. (9) with respect to $\phi$, we get the following equation of motion for the WIMP inside the neutron star:

$$
\frac{d^{2} \hat{u}}{d \phi^{2}}+\hat{u}=\frac{1}{J^{2}} \frac{M(r)}{M}+3 \frac{G M}{R} \frac{M(r)}{M} \hat{u}^{2}=\frac{1}{J^{2} \hat{u}^{3}}+\frac{3 G M}{R \hat{u}},
$$

where $\hat{u}=R u$ and again $J^{2}$ is measured in units of $G M R$. The initial conditions of this differential equation are $\hat{u}(\phi=0)=1$, which means that we have chosen $\phi=0$ at the point where the WIMP crosses the surface of the star. For the velocity

$$
\frac{d \hat{u}(\phi=0)}{d \phi}=\sqrt{\frac{2 G M}{R}-1+\frac{2}{J^{2}}} .
$$

The length of the path of the particle traveling inside the star is

$$
\frac{d l}{R}=\frac{d \phi}{\hat{u}^{2}} \sqrt{\left(\frac{d \hat{u}}{d \phi}\right)^{2}+\hat{u}^{2}} .
$$

We can find the length of the path of a particle inside the star if we integrate $\phi$ from zero up to the angle that $\hat{u}(\phi)=$ 1 again, which is the point where the particle exits from the star. Using Eqs. (14) and (17), and after having solved Eq. (15) numerically, and averaging over $J^{2}$ from 0 to 3.4 , we found that the average path inside the star is $1.87 R$ and $f=0.45 \sigma_{\chi} / \sigma_{\text {crit }}$. We should emphasize that the above estimate of $f$ holds for $\sigma_{\chi}<\sigma_{\text {crit }}$. It is understood that if $\sigma_{\chi}>\sigma_{\text {crit }}, f$ increases, saturating to 1 as soon as $\sigma_{\text {crit }}$ becomes larger than roughly $10^{-45} \mathrm{~cm}^{2}$. In addition, we should mention that our estimate is a lower bound for $f$. This is because in our derivation we assumed a 
constant density. Neutron stars are expected to be denser as we approach the center. In this case, $f$ will be larger than our estimate. To illustrate this, we examined the extreme case where all the mass of the star is concentrated at the core. In this scenario, although somewhat unrealistic, $f$ increases drastically because, for a large range of $J^{2}$, the orbits follow spirals around the center. A large fraction of the particles will be trapped in the star, not because of energy loss due to a collision, but due to the fact that they are trapped gravitationally. Our expectation is that the real case should be somewhere in the middle, and therefore we consider our previous estimate for $f$ as a lower bound.

Using Eq. (11) and the values for our typical neutron star, $M=1.4 M_{\odot}, R=10 \mathrm{~km}$, we get the rate of accretion of dark matter inside the star in particles per second,

$$
\mathcal{F}=\frac{3.042 \times 10^{25}}{m_{\chi}(\mathrm{GeV})} \times A \times f,
$$

where $A$ is a constant that parametrizes the local dark matter density in the vicinity of the neutron star in units of $0.3 \mathrm{GeV} / \mathrm{cm}^{3}$ (which is the standard dark matter density around the Earth). For cross sections $\sigma_{\chi}>10^{-45} \mathrm{~cm}^{2}$, $f=1$; otherwise $f$ is given by $f=0.45 \sigma_{\chi} / \sigma_{\text {crit }}$.

\section{ANNIHILATION RATE}

Once the WIMP undergoes one scattering inside the star, it loses, on average, enough energy to be captured by the star. Even if the kinetic energy is sufficient enough to make it exit from the star, it will be forced to return and probably scatter again, losing even more energy. The WIMP can repeat this process several times until its kinetic energy reduces down to the thermal velocity inside the star. It is easy to show that, for most cases of interest, the WIMP thermalizes very fast compared to the other time scales of the problem. We can make a very rough estimate of how long it takes for a WIMP moving with the average velocity of $270 \mathrm{~km} / \mathrm{sec}$ to obtain a velocity comparable to the thermal velocity. Let us assume that the WIMP has undergone one scattering and therefore has lost, on average, energy of $\left(2 m_{n} / m_{\chi}\right) v_{\text {esc }}^{2} / 2$, where $v_{\text {esc }}$ is the escape velocity from the star. A simple approximate calculation shows that it will take a few seconds before the WIMP intersects again with the star and again loses a fraction, $2 m_{n} / m_{\chi}$, of its energy. This depends on the cross section of the elastic collision between WIMP-nucleus, but as we showed in the previous section, for $\sigma_{\chi}>10^{-45} \mathrm{~cm}^{2}$, this will happen on average. In that case, it will take just a few hours before the kinetic energy of the WIMP reduces down to the thermal velocity. So our conclusion is that, for not extremely small cross sections, captured WIMPs will thermalize pretty fast and they will have a Maxwell-Boltzmann distribution in velocity and in distance from the center of the star.

The population of dark matter WIMPs inside the star is governed, in principle, by three processes. The first one is the accretion of WIMPs onto the star. The second is the evaporation, and the third one is the annihilation. Once the WIMPs thermalize inside the star, they follow a MaxwellBoltzmann distribution in velocity. Particles that are in the tail of the distribution (with large velocities) can escape from the star, if the velocity is larger than the escape velocity of the star. However, in the cases we are interested in, this process is exponentially suppressed. The rate of evaporating particles is proportional to $\exp \left(-G M m_{\chi} / R T\right)$ [18]. Since the radius of a neutron star is very small, and we are interested in WIMPs with mass of the order of $\mathrm{TeV}$, the rate becomes negligible. For a temperature of $100 \mathrm{keV}$ (which corresponds to a typical temperature of a neutron star that is a few thousand years old), and for $m_{\chi}=1 \mathrm{TeV}$, the suppression is $\exp \left(-10^{7}\right)$. Therefore we can safely ignore the evaporation process.

If the WIMP is a Majorana particle, for example, a Majorana neutrino, it is possible to coannihilate with another one. The annihilation depends on the cross section as well as the density of the WIMPs inside the star. The annihilation cross section should not be confused with the elastic cross section between WIMP-nucleus that was mentioned before. One big difference between the two is that the annihilation cross section for Majorana particles is usually velocity dependent. If the WIMP is a Majorana neutrino, it has an elastic cross section with nuclei

$$
\sigma_{\chi}=\frac{2 G_{F}^{2}}{\pi} \mu^{2} I_{s}
$$

where $\mu$ is the reduced mass of the system WIMP-nuclei and $I_{s}$ is a form factor that depends on the nucleus [19]. The annihilation cross section of two Majorana neutrinos depends on what channels are open for annihilation. If the mass of the Majorana neutrinos is larger than $100 \mathrm{GeV}$, the dominant channel is the annihilation to a pair of $W^{+}-W^{-}$ mediated by a $Z$ boson [20]. In the case where $m_{\chi} \gg$ $100 \mathrm{GeV}$, the annihilation cross section is given by

$$
\sigma_{A}=\frac{G_{F}^{2} m_{\chi}^{2}}{3 \pi} \beta^{2}
$$

where $\beta$ is the velocity of the WIMP (at the center of mass frame). Once the WIMP gets thermalized in the star, $\left\langle\beta^{2}\right\rangle=3 T /\left(2 m_{\chi}\right)$ ( $T$ being the temperature in the star).

The annihilation rate of WIMPs in the star is given by

$$
\Gamma_{A}=\left\langle\sigma_{\chi} v\right\rangle \int n^{2} d V
$$

where $\left\langle\sigma_{\chi} v\right\rangle$ is the thermally averaged annihilation cross section times the velocity and $n$ is the density of the WIMPs inside the neutron star. For convenience we shall assume that the density is constant inside the star. In this case, the population of WIMPs inside the star is governed by 


$$
\frac{d N}{d t}=\mathcal{F}-C_{A} N^{2}
$$

The constant $C_{A}=\left\langle\sigma_{\chi} v\right\rangle / V$, where $V$ is the volume of the star. The accretion rate $\mathcal{F}$ was derived in the previous section. The solution of Eq. (22) is

$$
N(t)=\sqrt{\frac{\mathcal{F}}{C_{A}}} \tanh \left(\frac{t}{\tau}\right) .
$$

The time scale $\tau=1 / \sqrt{\mathcal{F} C_{A}}$. The released energy due to the annihilation is

$$
E=C_{A} N^{2} m_{\chi}=\mathcal{F} \tanh ^{2}(t / \tau) m_{\chi} .
$$

The amount of the released energy depends on the time scale $\tau$. If $\tau$ is large compared to the age of the known neutron stars, the hyperbolic tangent is suppressed and the effect of the dark matter on the temperature of the star is negligible. For particles with a velocity independent annihilation cross section, $\tau$ is given by

$$
\tau=\frac{2.1 \times 10^{3} \text { years }}{\sqrt{\frac{A f \sigma_{39}}{m_{\chi}}}},
$$

where $\sigma_{39}$ is defined through $\left\langle\sigma_{A} v\right\rangle=\sigma_{39} 10^{-39} \mathrm{~cm}^{2}$. In the case of a Majorana particle, Eq. (23) holds only approximately because the cross section is velocity dependent, and therefore in conditions of thermal equilibrium, it is also temperature dependent. Since the temperature of the star changes as a function of time, this means that there is additional time dependence on the annihilation rate. Generally, for a Majorana neutrino with mass larger than $100 \mathrm{GeV}$, the cross section is given by Eq. (20) times a factor $\sin ^{4} \theta$ which denotes the suppression of the cross section due to a mixing between the left-handed neutrino (that interacts weakly) and a sterile right-handed neutrino $[10,20]$. The time scale $\tau$ is

$$
\tau=\frac{1}{\sqrt{\mathcal{F} C_{A}}}=\frac{5.98 \times 10^{5} \text { years }}{\sqrt{\frac{A f \sigma_{39}}{m_{\chi}^{2}}\left(\frac{T}{10^{8}}\right)}},
$$

where the temperature $T$ is measured in Kelvin degrees and $\sigma_{39}$ is defined by the relation $\sigma_{A}=\sigma_{39} 10^{-39} \beta^{2} \mathrm{~cm}^{2}$. The mass $m_{\chi}$ is measured in GeV. Equation (26) can be written more conveniently in terms of the mixing angle $\sin \theta$ as

$$
\tau=\frac{2.52 \times 10^{5} \text { years }}{\sqrt{A f \sin ^{4} \theta\left(\frac{T}{10^{8}}\right)}} .
$$

If the Majorana neutrino is exclusively left handed, $\sin \theta=$ 1 , and for a temperature of $10^{8} \mathrm{~K}$, the time scale is about $10^{5}$ years (depending on how much larger the local dark matter density is in the vicinity of the star compared to the one of the Earth). As it can be seen from (24), the annihilation saturates to $\mathcal{F}$ for times larger than roughly $3 \tau$. As we already mentioned, since the temperature of the star changes in time, Eqs. (26) and (27) are approximate. We shall return to the question of how fast the annihilation rate reaches the saturated value in the next section.

\section{COOLING AND HEATING THE NEUTRON STAR}

In this section we investigate the influence of the WIMP annihilation on the temperature of the neutron star. Naively, one would expect that such an effect should be negligible due to the fact that the accretion of dark matter represents a tiny fraction of the whole mass of the star. However, there are two elements that make this investigation interesting. The first one is that, although the trapped dark matter represents a small fraction of the mass of the star, the annihilation of two WIMPs releases a huge amount of energy. After the annihilation, this energy is carried mostly by leptons, quarks, and photons. Since they cannot escape from the star, they will heat it up. A small portion of the energy will be carried by neutrinos that will escape and will not contribute to the heating of the star. However, to first approximation, the energy carried by the neutrinos is negligible compared to the one carried by quarks, leptons, and photons. So, we are going to assume that the whole annihilation energy will not be carried by neutrinos, and therefore this energy will heat up the star. The second reason we investigate this effect is that the energy released by the annihilation and, consequently, the emissivity of this process does not scale with the temperature. As long as the equilibrium between accretion and annihilation has been reached, the released energy per time remains unchanged. All the dominant cooling processes of a neutron star scale with positive powers of $T$. This means that inevitably, even if the emissivity due to WIMP annihilation is small, as the temperature of the star decreases, the WIMP annihilation emissivity will dominate at some point.

Let us assume for the moment, and we shall examine later under what conditions this is possible, that the time $3 \tau$ has been reached and the released energy from the annihilation of the WIMPs is $E=\mathcal{F} m_{\chi}$. The emissivity, i.e. released energy per volume per time, is

$$
\epsilon_{\mathrm{dm}}=\frac{E}{4 \pi R^{3} / 3}=\frac{3 \mathcal{F} m_{\chi}}{4 \pi R^{3}}=A 1.16 \times 10^{4} \mathrm{erg} \mathrm{cm}^{-3} \mathrm{~s}^{-1} .
$$

There are several processes that contribute to the cooling of a neutron star, depending on the form of matter, the temperature, and the density of the star. If the star is sufficiently dense, unpaired quark matter or other exotic phases might occur deep inside the star. If the neutron star has unpaired quark matter, for the first million years it cools very fast due to neutrino emission via the direct Urca process. In this case the emissivity scales as $\epsilon_{\nu} \sim$ $T^{6}$. Direct Urca processes are allowed in sufficiently dense nuclear matter, nuclear matter with pion condensation, 
kaon condensation, or nonzero hyperon density, and in all phases of quark matter except the Color Flavor Locked phase; see [21] and references therein. For neutron stars that are not in sufficiently high density, direct Urca processes $n \rightarrow p+e+\bar{\nu}, p+e \rightarrow n+\nu$ are kinematically forbidden. In this case, a bystander neutron is needed in order to kinematically assist the reaction. This is the socalled modified Urca process. During the epoch dominated by the modified Urca, the star loses energy through neutrino emission, by converting protons and electrons to neutrons and vice versa. The emissivity of this process scales as $\sim T^{8}$. It is [22]

$$
\epsilon_{\nu}=\left(1.2 \times 10^{4} \mathrm{erg} \mathrm{cm}^{-3} \mathrm{~s}^{-1}\right)\left(\frac{n}{n_{0}}\right)^{2 / 3}\left(\frac{T}{10^{7} \mathrm{~K}}\right)^{8},
$$

where $n$ is the baryon density of the star and $n_{0}=$ $0.17 \mathrm{fm}^{-3}$ is the baryon density in nuclear matter. In our calculation for the neutron star of $M=1.4 M_{\odot}$ and $R=$ $10 \mathrm{~km}$, we are going to use the average density $n=3.9 \times$ $10^{38}$ particles per $\mathrm{cm}^{3}$ and therefore $n / n_{0}=2.3$.

After the first million years and, roughly, as soon as the temperature of the star drops below $10^{8} \mathrm{~K}$, the dominant mechanism of cooling is not through neutrino emission anymore, but through photon emission from the surface of the star. The rate of heat loss from the surface of the star is

$$
L_{\gamma}=4 \pi R^{2} \sigma T_{\text {surface }}^{4}
$$

where $\sigma$ is the Stefan-Boltzmann constant and $T_{\text {surface }}$ is the temperature of the surface of the star. The surface of a neutron star is usually colder than the interior of the star. This change in the temperature occurs inside the crust of the neutron star, taking place within 100 meters below the surface. The surface temperature of the star is well approximated by [23-25]

$$
T_{\text {surface }}=\left(0.87 \times 10^{6} \mathrm{~K}\right)\left(\frac{g_{s}}{10^{14} \mathrm{~cm} / \mathrm{s}^{2}}\right)^{1 / 4}\left(\frac{T}{10^{8} \mathrm{~K}}\right)^{0.55},
$$

where $T$ is the interior temperature of the star and $g_{s}=$ $G M / R^{2}$ is the surface gravity. The rate of heat loss $L_{\gamma}$ can now be expressed in terms of the interior temperature as

$$
L_{\gamma}=4 \pi R^{2} \sigma\left(0.87 \times 10^{6} \mathrm{~K}\right)^{4}\left(\frac{g_{s}}{10^{14} \mathrm{~cm} / \mathrm{s}^{2}}\right)\left(\frac{T}{10^{8} \mathrm{~K}}\right)^{2.2} .
$$

If we divide $L_{\gamma}$ over the volume of the star, we can get an "effective" emissivity of photons measured in energy over volume and time,

$$
\epsilon_{\gamma}=\frac{L_{\gamma}}{(4 / 3) \pi R^{3}}=1.8 \times 10^{14}\left(\frac{T}{10^{8} \mathrm{~K}}\right)^{2.2} \mathrm{erg} \mathrm{cm}^{-3} \mathrm{~s}^{-1},
$$

where we used $g_{s}=1.85 \times 10^{14} \mathrm{~cm} / \mathrm{s}^{2}$.
In order to be able to derive the temperature as a function of time, we need to know the heat capacity of the star. For a gas of noninteracting fermions, the specific heat is given by [22]

$$
c_{V}=\frac{k_{B}^{2} T}{3 \hbar^{3} c} \sum_{i} p_{F}^{i} \sqrt{m_{i}^{2} c^{2}+\left(p_{F}^{i}\right)^{2}},
$$

where the sum runs over the different species. In the case we investigate, namely, the one of noninteracting nuclear matter, $i$ runs over $n, p, e$ and the Fermi momenta for neutral matter in weak equilibrium are

$$
\begin{gathered}
p_{F}^{n}=(340 \mathrm{MeV})\left(\frac{n}{n_{0}}\right)^{1 / 3}, \\
p_{F}^{p}=p_{F}^{e}=(60 \mathrm{MeV})\left(\frac{n}{n_{0}}\right)^{2 / 3} .
\end{gathered}
$$

The cooling of the star is dictated by the differential equation

$$
\begin{aligned}
\frac{d T}{d t} & =\frac{-L_{\nu}-L_{\gamma}+L_{\mathrm{dm}}}{V c_{V}}=\frac{V\left(-\epsilon_{\nu}-\epsilon_{\gamma}+\epsilon_{\mathrm{dm}}\right)}{V c_{V}} \\
& =\frac{-\epsilon_{\nu}-\epsilon_{\gamma}+\epsilon_{\mathrm{dm}}}{c_{V}},
\end{aligned}
$$

where the volume of the star $V$ drops out at the end. We have neglected the contribution of the WIMPs to the specific heat, since they consist of a tiny fraction of the mass of the star. We solved Eq. (37) numerically, by imposing an initial temperature for the star of $10^{10} \mathrm{~K}$ at very early time. However, we should emphasize that the temperature is very insensitive to the initial condition. It affects the temperature only during the first years of the star's life, but it has no effect later on. In Fig. 1, we have plotted in a logarithmic scale the internal and the surface temperature of the star as a function of time, starting from time $t=1000$ years, up to $100 \times 10^{6}$ years. We have plotted the temperature for three different cases that correspond to different local dark matter densities for the vicinity of the star. We chose the local dark matter density to be the one of the Earth $\left(0.3 \mathrm{GeV} / \mathrm{cm}^{3}\right), 10$ times larger and 100 times larger than the one of the Earth. For comparison, we also plotted the cooling curve of the same star without including the effect of dark matter annihilation. As we can see from the figures, the dark matter annihilation does not affect the temperature of the star up to $t=10 \times$ $10^{6}$ years. Between $10^{3}$ and $10^{6}$ years, the star cools due to neutrino emission via the modified Urca process, while after roughly $10^{6}$ years it cools through photon emission from its surface. However, by inspection of Eqs. (28), (29), and (33), we see that the dark matter annihilation emissivity scales with the lowest power of $T$. More precisely, $\epsilon_{\mathrm{dm}}$ does not depend on $T$, once equilibrium between the rate of accretion of dark matter and the rate of annihilation has been established. This means that inevitably, when the 


\section{Internal Temperature}

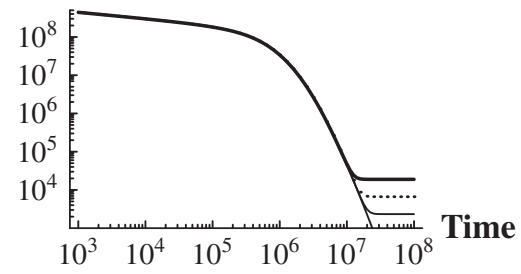

Surface Temperature

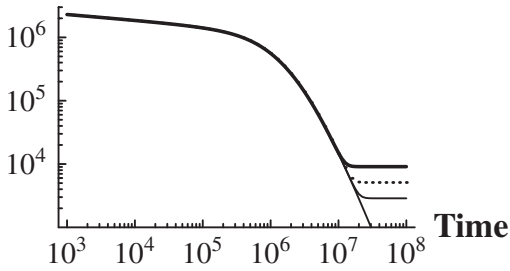

FIG. 1. Left panel: The internal temperature of a neutron star (in Kelvin) with $M=1.4 M_{\odot}$ and $R=10 \mathrm{~km}$ as a function of time (in years). The solid line that crosses the time axis corresponds to the case where the effect of dark matter annihilation is neglected. The thin solid line corresponds to a local dark matter density for the star of $0.3 \mathrm{GeV} / \mathrm{cm}^{3}$. The dashed and thick solid lines correspond to local densities of 3 and $30 \mathrm{GeV} / \mathrm{cm}^{3}$, respectively. Right panel: Same as in the left panel but for the surface temperature of the neutron star.

temperature of the star drops sufficiently, the power of the dark matter annihilation that heats up the star will equal the power of photon emission, and as a result the temperature will remain flat as a function of time. This happens roughly at $t=10 \times 10^{6}$ years and at surface temperatures between 3000 and $10000 \mathrm{~K}$ (depending on the local dark matter density, the mass, and the radius of the star). To entertain the possibility of having a neutron star with a local dark matter density 10 or 100 times larger than $0.3 \mathrm{GeV} / \mathrm{cm}^{3}$, we can use an indicative profile density for the dark matter halo. We consider the Navarro-Frenk-White profile, where the dark matter density is given by [26]

$$
\rho(r)=\frac{\rho_{0}}{\left(\frac{r}{R}\right)^{\gamma}\left(1+\left(\frac{r}{R}\right)^{\alpha}\right)^{(\beta-\gamma) / \alpha}} .
$$

This profile has a spike in the center of the Galaxy for positive $\gamma$. We shall use $\alpha=1, \beta=3, \gamma=1, R=$ $20 \mathrm{kpc}$, and $\rho_{0}=0.235 \mathrm{GeV} / \mathrm{cm}^{3}$. Given this density profile, a neutron star with density 10 and 100 times larger than the local dark matter density of the Earth should be 1.37 and $0.15 \mathrm{kpc}$ from the center of the Galaxy, respectively. The position of the Earth is roughly $8 \mathrm{kpc}$ from the center of the Galaxy. This means that a neutron star that exhibits a flatness in temperature of $\sim 10^{4} \mathrm{~K}$ for time $t>$ $10 \times 10^{6}$ years due to the dark matter annihilation should lie at least $\sim 6.5 \mathrm{kpc}$ away from the Earth. This limit can be improved, if the star has "more convenient" mass and radius than what we have considered for a typical neutron star. The emissivity $\epsilon_{\mathrm{dm}}$ is proportional to $\mathcal{F}$, which is proportional to the factor $M R /(1-2 G M / R)$. The emissivity $\epsilon_{\mathrm{dm}}$ is also proportional to the local dark matter density. When we quote results with local dark matter density 10 times larger than the one of the Earth, this does not imply per se that the star has to be at a region of the Galaxy with $\rho_{\mathrm{dm}}=3 \mathrm{GeV} / \mathrm{cm}^{3}$, but the factor $M R /(1-2 G M / R)$ times the local dark matter density should be 10 times the same factor for our typical star with $M=1.4 M_{\odot}$ and $R=10 \mathrm{~km}$ times the density of $0.3 \mathrm{GeV} / \mathrm{cm}^{3}$. For example, a neutron star of $M=2 M_{\odot}$ and $R=6 \mathrm{~km}$ gives a factor of $\sim 26.5$ compared to our typical star. Therefore our results for density 100 times the density of the Earth are applied also for a star with mass and radius given in the previous sentence and a local dark matter density of only $100 / 26.5=3.77$ times the density of dark matter around the Earth.

Now we return to a question we posed earlier. In the results we have presented, we have assumed that equilibrium between the accretion and the annihilation rate has taken place before $t=10 \times 10^{6}$ years, where the effect of the WIMP annihilation becomes important. If $\tau$ is much larger than $10 \times 10^{6}$ years, the annihilation rate has not reached the asymptotic value $\sim \mathcal{F}$ and the effect on the temperature of the star will be negligible. We saw in Fig. 1 that, even if the asymptotic annihilation rate is reached very early, the WIMP annihilation does not affect the temperature for $t<10 \times 10^{6}$ years (or equivalently for temperatures higher than $10^{4} \mathrm{~K}$ ). This means that, up to $10^{7}$ years, the temperature of the star is controlled by modified Urca and photon emission. We already mentioned that, if the WIMP annihilation cross section is velocity dependent (like in the case of Majorana neutrinos), Eqs. (24), (26), and (27) hold only approximately since $\tau$ is temperature dependent (and implicitly time dependent). In particular, Eqs. (26) and (27) can give a lower bound estimate of how fast the asymptotic annihilation rate is reached. For most candidates of our interest, Majorana neutrinos or Majorana technibaryons [10], we can safely take $f=1$, since the elastic cross section with nuclei is not smaller than $10^{-45} \mathrm{~cm}^{2}$. By using Eq. (27), with $f=1$, $A=10$, and an "average" temperature of $10^{8} \mathrm{~K}$ for the star, we get $\tau \sim 10^{6}$ years for cross section suppression $\sin \theta>0.1$. In reality, the situation is better, because this estimate is done assuming constant $T=10^{8} \mathrm{~K}$. However, the temperature of the star is much higher at the beginning ( $\sim 10^{10} \mathrm{~K}$ ), and due to the modified Urca process, it drops down to $T=10^{8} \mathrm{~K}$ roughly at $t=10^{6}$ years. The higher temperature at the beginning brings the annihilation rate to the asymptotic value more quickly. In addition, for $10^{6}<$ $t<10^{7}$ years, where the star cools mainly due to photon emission, the annihilation rate can also improve towards the asymptotic value, although since the temperature falls really fast, this happens at a slow rate. For the two cases we 
mentioned, Majorana neutrinos and Majorana technibaryons, the annihilation rate reaches the asymptotic value before $10 \times 10^{6}$ years. For example, for a Majorana neutrino, Eq. (27), for $\sin \theta=1, A=100$, and $f=1$, gives $2.52 \times 10^{4}$ years (for $T=10^{8} \mathrm{~K}$ ). For a Majorana neutrino (or Majorana technibaryon) with suppressed coupling to the $Z$ boson in order to account for the right dark matter density, for a mass of $1 \mathrm{TeV}, \sin \theta=0.26$ [10]. In this case, Eq. (27) gives a characteristic time scale $\tau=3.7 \times$ $10^{5}$ years, with $A, f$, and $T$ as before. Therefore, for most cases of interest and unless the cross section is very small, the annihilation rate reaches the accretion rate before $10 \times 10^{6}$ years, which is the time where the dark matter annihilation affects the temperature of the neutron star.

\section{CONCLUSIONS}

We investigated the effect of WIMP annihilation on the temperature of a neutron star. We found that, for a typical neutron star with a local dark matter density at least $3 \mathrm{GeV} / \mathrm{cm}^{3}$, and if the WIMP has an elastic cross section not smaller than $10^{-46} \mathrm{~cm}^{2}$, the WIMP annihilation flattens out the temperature of the star around $\sim 10^{4} \mathrm{~K}$ at $t=$ $10 \times 10^{6}$ years. Two neutron stars that have different local dark matter densities would have different final temperatures scaling as $T \sim \rho_{\mathrm{dm}}^{1 / 2.2}$. Given the uncertainty in our knowledge of the age of a neutron star and the fact that the peak of a blackbody spectrum of $10^{4} \mathrm{~K}$ lies in the infrared, it is a challenge to observe such an effect, which would possibly be a signature of WIMP annihilation. Alternatively, instead of trying to spot neutron stars with such a low temperature, it might be more efficient to study pulsars, detected already by their nonthermal emission and to constrain their thermal emission putting an upper bound on their temperature.

In our analysis, we disregarded the interesting possibility of having an exotic phase of quark matter in the typical neutron star. Exotic quark matter phases like color superconductivity can have an effect on the cooling of a neutron star [27-30]. This could change the dominant cooling process for the first million years, but it is highly unlikely that our conclusions would change regarding the effect of the WIMP annihilation. We also neglected reheating mechanisms that could possibly be present for old neutron stars. These mechanisms can be viscous dissipation of rotational energy within the star [31], energy release due to weak deviations from beta equilibrium [32], accretion from interstellar gas, or others; see [33] and references therein. All of these mechanisms are model dependent, and it is not clear what their effect is on the temperature of an old neutron star compared to the WIMP annihilation mechanism. As a rule, in a nonsuperfluid old star without a magnetic field and an accreted envelope, the alternative mechanisms mentioned above probably cannot prevent the star from getting a very low temperature. In this case, WIMP annihilation might be the dominant reheating mechanism of the star.

Much theoretical work remains to be done. An interesting question is if the effect of WIMP annihilation inside a neutron star can change the suggested mechanisms for superbursts and gravitational wave bursts proposed in [34-36]. These mechanisms assume a phase transition to quark matter at the center of the neutron star via metastable phases, which can lead to a burst. The released energy from the WIMP annihilation inside the star might work as a catalyst, accelerating the phase transition and making it impossible for the metastable phases to persist.

\section{ACKNOWLEDGMENTS}

I am grateful to Mark Alford, Maxim Khlopov, and Krishna Rajagopal for their comments. The work of C. K. is supported by the Marie Curie Excellence Grant under Contract No. MEXT-CT-2004-013510.
[1] D. N. Spergel et al. (WMAP Collaboration), Astrophys. J. Suppl. Ser. 170, 377 (2007).

[2] C. Alcock et al. (MACHO Collaboration), Astrophys. J. 542, 281 (2000).

[3] G. Jungman, M. Kamionkowski, and K. Griest, Phys. Rep. 267, 195 (1996).

[4] G. Bertone, D. Hooper, and J. Silk, Phys. Rep. 405, 279 (2005).

[5] F. Sannino and K. Tuominen, Phys. Rev. D 71, 051901 (2005).

[6] D. K. Hong, S. D. H. Hsu, and F. Sannino, Phys. Lett. B 597, 89 (2004).

[7] D. D. Dietrich, F. Sannino, and K. Tuominen, Phys. Rev. D 72, 055001 (2005).
[8] S. B. Gudnason, C. Kouvaris, and F. Sannino, Phys. Rev. D 73, 115003 (2006).

[9] S. B. Gudnason, C. Kouvaris, and F. Sannino, Phys. Rev. D 74, 095008 (2006).

[10] C. Kouvaris, Phys. Rev. D 76, 015011 (2007).

[11] W. H. Press and D. N. Spergel, Astrophys. J. 296, 679 (1985).

[12] A. Gould, Astrophys. J. 321, 571 (1987).

[13] A. Gould, Astrophys. J. 328, 919 (1988).

[14] Y. B. Zeldovich, A. A. Klypin, M. Y. Khlopov, and V. M. Chechetkin, Yad. Fiz. 31, 1286 (1980) [Sov. J. Nucl. Phys. 31, 664 (1980)].

[15] D. S. Akerib et al. (CDMS Collaboration), Phys. Rev. Lett. 93, 211301 (2004). 
[16] I. Goldman and S. Nussinov, Phys. Rev. D 40, 3221 (1989).

[17] P. A. Collins, R. Delbourgo, and R. M. Williams, J. Phys. A 6, 161 (1973).

[18] L. M. Krauss, M. Srednicki, and F. Wilczek, Phys. Rev. D 33, 2079 (1986).

[19] J. D. Lewin and P. F. Smith, Astropart. Phys. 6, 87 (1996).

[20] K. Enqvist and K. Kainulainen, Phys. Lett. B 264, 367 (1991).

[21] M. Alford, P. Jotwani, C. Kouvaris, J. Kundu, and K. Rajagopal, Phys. Rev. D 71, 114011 (2005).

[22] S. L. Shapiro and S. A. Teukolsky (Wiley, New York, 1983), p. 645.

[23] E.H. Gundmundsson, C.J. Pethick, and R.I. Epstein, Astrophys. J. 259, L19 (1982).

[24] E.H. Gundmundsson, C. J. Pethick, and R. I. Epstein, Astrophys. J. 272, 286 (1983).

[25] D. Page, J. M. Lattimer, M. Prakash, and A. W. Steiner, arXiv:astro-ph/0403657.
[26] J. F. Navarro, C. S. Frenk, and S. D. M. White, Astrophys. J. 490, 493 (1997).

[27] M. G. Alford, K. Rajagopal, and F. Wilczek, Phys. Lett. B 422, 247 (1998).

[28] M. G. Alford, K. Rajagopal, and F. Wilczek, Nucl. Phys. B537, 443 (1999).

[29] K. Rajagopal and F. Wilczek, arXiv:hep-ph/0011333.

[30] M. Alford, Prog. Theor. Phys. Suppl. 153, 1 (2004).

[31] A. Alpar, W. Brinkmann, H. Ogelman, U. Kiziloglu, and D. Pines, Astron. Astrophys. 177, 101 (1987).

[32] A. Reisenegger, Astrophys. J. 442, 749 (1995).

[33] D. G. Yakovlev and C.J. Pethick, Annu. Rev. Astron. Astrophys. 42, 169 (2004).

[34] D. K. Hong, S. D. H. Hsu, and F. Sannino, Phys. Lett. B 516, 362 (2001).

[35] Z. Berezhiani, I. Bombaci, A. Drago, F. Frontera, and A. Lavagno, Astrophys. J. 586, 1250 (2003).

[36] L. M. Lin, K.S. Cheng, M. C. Chu, and W. M. Suen, Astrophys. J. 639, 382 (2006). 